\title{
Alterations in the basal ganglia in patients with brain tumours may be due to excessive iron deposition
}

\author{
VÍT HERYNEK ${ }^{1}$, DITA WAGNEROVÁ ${ }^{1}$, ALBERTO MALUCELLI ${ }^{2}$, \\ JOSEF VYMAZAL ${ }^{3}$, MARTIN SAMEŠ ${ }^{2}$ and MILAN HÁJEK ${ }^{1}$ \\ ${ }^{1}$ Department of Diagnostic and Interventional Radiology, Institute for Clinical and Experimental Medicine, Vídeňská, \\ Prague 14021; ${ }^{2}$ Department of Neurosurgery, JE Purkyně University and Masaryk Hospital, Sociální péče, \\ Ústí nad Labem 40113; ${ }^{3}$ Department of Radiology, Na Homolce Hospital, Roentgenova, Prague 15030, Czech Republic
}

Received February 17, 2014; Accepted September 26, 2014

DOI: $10.3892 / \mathrm{ol} .2014 .2638$

\begin{abstract}
The accumulation of iron in the brain is a common physiological process. However, alterations in the deposition of iron or other paramagnetic substances are associated with various diseases. In the present study, the deposition of paramagnetic substances in patients with brain tumours was evaluated using $\mathrm{T}_{2}$ relaxometry. A total of 23 patients with untreated tumours or with recurrent tumours following treatment, together with a group of 19 age-matched healthy controls, were examined using $\mathrm{T}_{2}$ relaxometry at $3 \mathrm{~T}$. The relaxation times in the basal ganglia, thalamus and white matter were evaluated. Significantly lower $T_{2}$ relaxation times were identified in the basal ganglia and thalamus of the patients with tumours, as compared with those of the controls $(\mathrm{P}<0.05)$. No statistically significant difference was identified between patients with untreated or recurrent brain tumours. The reduction in $\mathrm{T}_{2}$ relaxation times in the brain tumour patients was possibly caused by the accumulation of iron, since iron homeostasis is known to be altered in patients with tumours. We propose that increased iron deposition is a consequence of a higher risk of oxidative stress caused by an increased iron concentration in the plasma or cerebrospinal fluid.
\end{abstract}

\section{Introduction}

The accumulation of non-haemic iron in the brain, particularly in the basal ganglia (BG), is a common process initiated shortly following birth (1). During the first two decades of life, the iron concentration in the $\mathrm{BG}$ increases rapidly and then the accumulation slows down, and the concentration of

Correspondence to: Dr Vít Herynek, Department of Diagnostic and Interventional Radiology, Institute for Clinical and Experimental Medicine, Vídeňská 1958/9, Prague 14021, Czech Republic

E-mail: vit.herynek@medicon.cz

Key words: magnetic resonance imaging, brain tumours, basal ganglia, iron, oxidative stress iron in the BG follows an approximately exponential association curve. Alterations in the deposition of iron and other paramagnetic substances have been associated with various diseases. Increased deposition of paramagnetic substances in the brain has been observed in patients with liver diseases (2), psychiatric (3) and neurodegenerative disorders (4,5).

Although iron is an essential element in human nutrition, free iron $\left(\mathrm{Fe}^{2+}\right)$ is closely associated with the production of reactive oxygen species (ROS), which may induce biological damage through oxidative stress. In the Fenton reaction, $\mathrm{Fe}^{2+}$ reduces $\mathrm{H}_{2} \mathrm{O}_{2}$ and subsequently produces a hydroxyl radical $(\bullet \mathrm{OH})$. Increased iron deposition in ferritin molecules is therefore considered to be a protective mechanism. The $\mathrm{Fe}^{2+}$ ions are trapped by ferritin, oxidised to $\mathrm{Fe}^{3+}$ and safely stored inside the protein molecule.

However, increased iron stores may be associated with an elevated risk of cancer. The association between iron and carcinogenesis has been recognised for a number of years (6). Increased body iron stores (indicated by higher serum ferritin levels and elevated transferrin concentrations) have been associated with malignant neoplasms (7) and leukaemia (8). Animal experiments as early as 1959 revealed that repeated injections of iron induce malignant tumours (9). Iron may be involved in the initiation or promotion of colorectal, liver, kidney, lung, stomach and other types of cancer (10).

In addition, increased concentrations of an iron-storage protein, ferritin, have been detected in the cerebrospinal fluid (CSF) of patients with glioblastoma (11). The authors ascertained the presence of ferritin in tumour cells in one patient and hypothesised that CSF ferritin was secreted by the tumour cells. The transfer of ferritin from the serum through the blood-CSF barrier does not occur on a large scale, due to the high molecular weight of ferritin and its hydrodynamic radius.

Attempts have been made to reduce the impact of iron redox activity during cancer treatment through the chelating of surplus iron ions (12); however, the alterations in Fe metabolism in cancer are not yet fully understood. Changes may occur during iron absorption, iron transport and iron storage $(7,8,11,12)$. Tumour cells are also known for the upregulation of transferrin receptor expression on the cell surface (12). 
Magnetic resonance imaging (MRI) is sensitive to the presence of paramagnetic ions in tissues. Alterations in paramagnetic or superparamagnetic ion concentrations manifest as a hyperintense signal on $\mathrm{T}_{1}$-weighted images or as a hypointense signal on $\mathrm{T}_{2}$-weighted images, as the ions substantially shorten relaxation times. Iron in iron-storage molecules substantially shortens $\mathrm{T}_{2}$ due to its magnetic properties. The most common type of iron-storage molecule, ferritin, which is soluble, also exerts a clear effect on the $T_{1}$ relaxation time, whereas insoluble pathological hemosiderin markedly influences $\mathrm{T}_{2}$, but has no significant effect on $\mathrm{T}_{1}(13)$. The changes in relaxation times may be quantified by MR relaxometry (14).

In the present study, the deposition of paramagnetic substances in patients with brain tumours was retrospectively evaluated using $\mathrm{T}_{2}$ relaxometry.

\section{Materials and methods}

Patients. A total of 23 patients (mean age $46 \pm 12$ years) were examined at the Department of Diagnostic and Interventional Radiology, Institute for Clinical and Experimental Medicine (Vídeňská, Czech Republic). The patients were divided into two groups. Group 1 consisted of 12 subjects (mean age, $46 \pm 13$ years) with an untreated tumour in the brain: Six subjects with high-grade gliomas (HGG), four subjects with low-grade gliomas (LGG) and two subjects with lymphomas (Lym). Group 2 consisted of 11 subjects (mean age, 46 \pm 11 years) with tumour recurrence following treatment (chemotherapy and radiotherapy and/or resection). The primary tumour was HGG in six subjects and LGG in five subjects. All patients involved in the study had the diagnosis verified by histological methods. The control group consisted of 19 age-matched healthy patients (mean age, $47 \pm 12$ years). All patients were informed with regard to the examination procedure and provided an informed consent approved by the local ethical committee. Clinical procedures were certified according to the ISO 9001:2008 norm.

MRI. A 3 Tesla clinical MR imager Magnetom Trio (Siemens, Erlangen, Germany) equipped with a transmit/receive (Tx/Rx) head coil was used. A standard imaging procedure consisting of native $\mathrm{T}_{1^{-}}$and $\mathrm{T}_{2}$-weighted images and contrast-enhanced $\mathrm{T}_{1}$-weighted images (in the patient group only) was supplemented with a Carr Purcell Meiboom Gill sequence (32 echoes, echo-spacing echo time $=13.2 \mathrm{~ms}$, repetition time $=3,000 \mathrm{~ms}$ and slice thickness $=5 \mathrm{~mm}$ ) for $\mathrm{T}_{2}$-mapping. A slice containing the $\mathrm{BG}$ was evaluated. $\mathrm{T}_{2}$ maps were calculated using custom-made ViDi software (Department of Diagnostic and Interventional Radiology, Institute for Clinical and Experimental Medicine) utilising a three-parameter fit (15). $\mathrm{T}_{2}$ values were then obtained from the globus pallidus (GP), putamen (Put), caudate nucleus (CN), thalamus (Th) and frontal white matter (WM) in the two hemispheres. However, tumours are commonly accompanied by extensive oedema, which may significantly prolong the $\mathrm{T}_{2}$ relaxation time in BG. This influence in the ipsilateral hemisphere cannot be separated from other changes that affect $\mathrm{T}_{2}$ relaxation, thus, the $T_{2}$ values were substantially dispersed. Therefore, for further evaluation and comparison, only values found in the contralateral hemisphere were used (Fig. 1). Infiltration of the

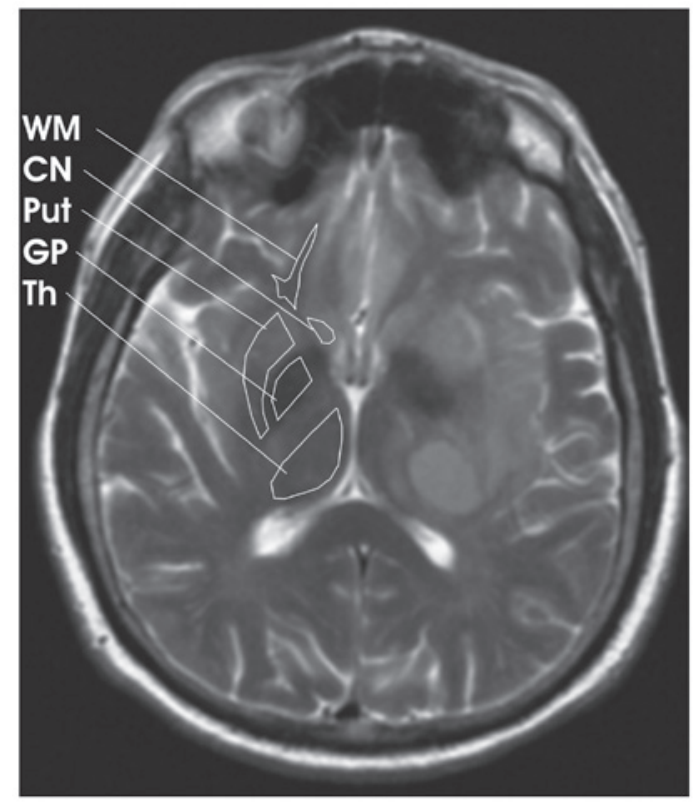

Figure 1. $\mathrm{A}_{2}$-weighted magnetic resonance image of a patient with a high-grade glioma IV (glioblastoma multiforme in the left hemisphere). The regions of interest in the white matter (WM), caudate nucleus $(\mathrm{CN})$, putamen (Put), globus pallidus (GP) and thalamus (Th) contralateral to the lesion are highlighted.

tumours into the contralateral hemisphere was not observed in the selected patients.

Statistical analysis. Student's t-test was used for comparing the relaxation times of the different patient groups and the controls. $\mathrm{P}<0.05$ was considered to indicate a statistically significant difference. The equality of variances was analysed using an F-test. For unequal variances, a variant of the t-test for unequal variances was employed; this was the case for data obtained from the WM.

\section{Results}

$T_{2}$ relaxation times in patients with untreated and recurrent, treated brain tumours. Significantly lower $\mathrm{T}_{2}$ relaxation times were identified in the GP, Put, $\mathrm{CN}$ and Th of brain tumour patients as compared with those of the controls $(\mathrm{P}<0.05$, Table I). In the group of patients with untreated brain tumours, the $\mathrm{T}_{2}$ in the GP, CN and Th was significantly reduced, as compared with the controls $(\mathrm{P}<0.05)$. However, the difference in the Put $\mathrm{T}_{2}$ values was not statistically significant due to the high data dispersion. In the patients with recurrent tumours who had received treatment, significantly lower $T_{2}$ values in the GP, Put, CN, Th and WM were detected, as compared with the controls $(\mathrm{P}<0.05)$.

No statistically significant differences were identified between patients with untreated tumours and those with recurrent tumours in any of the examined structures. However, a trend toward lower $T_{2}$ values in the patients with recurrent tumours was detected, which was verified by a paired t-test between the average values in the given structures in the two patient groups.

No difference in the $\mathrm{WM} \mathrm{T}_{2}$ values between the group of patients with untreated tumours and the control group was 
Table I. $\mathrm{T}_{2}$ relaxation times in the globus pallidus, putamen, caudate nucleus, thalamus and white matter of patients (contralateral to the lesion) and controls.

$\mathrm{T}_{2}(\mathrm{~ms})$

\begin{tabular}{lccccc}
\cline { 2 - 5 } Patient group & Globus pallidus & Putamen & Caudate nucleus & Thalamus & White matter \\
\hline All patients & $51.4 \pm 2.2^{\mathrm{a}}$ & $63.2 \pm 4.1^{\mathrm{a}}$ & $73.4 \pm 4.8^{\mathrm{a}}$ & $70.8 \pm 2.7^{\mathrm{a}}$ & $69.2 \pm 4.6$ \\
Patients with an untreated tumour & $52.0 \pm 2.0^{\mathrm{a}}$ & $63.5 \pm 3.8$ & $74.0 \pm 5.0^{\mathrm{a}}$ & $70.6 \pm 2.4^{\mathrm{a}}$ & $70.3 \pm 5.3$ \\
Patients with a recurrent tumour & $50.9 \pm 2.3^{\mathrm{a}}$ & $62.8 \pm 4.3^{\mathrm{a}}$ & $72.9 \pm 4.5^{\mathrm{a}}$ & $71.0 \pm 3.0^{\mathrm{a}}$ & $68.0 \pm 3.3^{\mathrm{a}}$ \\
Controls & $55.2 \pm 2.0$ & $67.3 \pm 4.7$ & $78.4 \pm 3.4$ & $75.1 \pm 2.2$ & $70.5 \pm 2.5$ \\
\hline
\end{tabular}

${ }^{\mathrm{a}} \mathrm{P}<0.05$, as compared with the control group.

observed; however, a significant difference in these values between the group of patients with recurrent tumours and the control group was identified $(\mathrm{P}<0.05)$.

$T_{2}$ relaxation times in patients with different types of brain tumour. The graph in Fig. 2 shows the distribution of $\mathrm{T}_{2}$ values of GP in all investigated groups. The two patients with Lym are presented separately. No statistically significant differences were identified between subjects with different grades of newly diagnosed gliomas (HGG and LGG) or those with Lym. However, the numbers of subjects in these subgroups were too low for meaningful statistical analysis.

\section{Discussion}

MRI is an in vivo imaging method sensitive to the paramagnetic ion content in tissues. In addition to $T_{2}$ mapping, other potentially more sensitive methods, such as $\mathrm{T}_{2}{ }^{*}$ mapping or susceptibility weighted imaging (SWI), may be of interest and may be examined in a prospective study. However, the length of the procedures in the present study did not permit the addition of these sequences. Although the $\mathrm{T}_{2}$ relaxation time reflects the tissue structure and the water content, in addition to the presence of paramagnetic ion, which renders interpretation more difficult, this measurement is less sensitive to possible artefacts resulting from external field heterogeneities than $\mathrm{T}_{2}{ }^{*}$ mapping or SWI, and is a robust and effective technique. In addition, $\mathrm{T}_{2}$ mapping was included in our standard imaging procedure and the majority of the subjects were evaluated retrospectively, therefore alterations to the method were not possible.

The reduction in the $\mathrm{T}_{2}$ relaxation time in the $\mathrm{BG}$ observed in the present study may be caused by paramagnetic ion accumulation. A similar effect (i.e. $\mathrm{T}_{2}$ shortening) has been observed in a range of psychiatric and neurodegenerative diseases, and has been predominantly associated with iron accumulation $(3,16)$. The trend detected in the present study towards lower $T_{2}$ values in the patients with recurrent tumours, as compared with the patients with newly diagnosed lesions, may indicate the worsening of the patient's state with time and disease progression, or the possible further damage to the tissue caused by radio/chemotherapy.

Although relaxometry is completely unspecific, the element responsible for $\mathrm{T}_{2}$ shortening is hypothesised to be iron, which

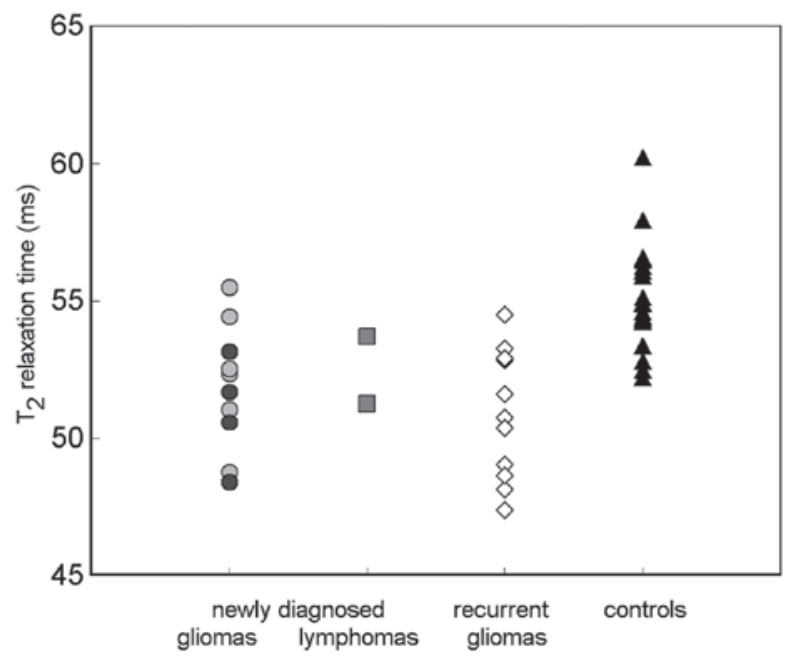

Figure 2. Distribution of $\mathrm{T}_{2}$ values in the contralateral globus pallidus in patients with newly diagnosed gliomas (high-grade glioma, light grey $\bullet$; low-grade glioma, dark grey $\bullet$ ), lymphomas (ロ) and recurrent gliomas $(\diamond)$, and controls $(\mathbf{\Delta})$

is closely associated with cancer initiation $(6,10)$ and tumour growth (17). Iron, an essential element for human life, may also be a carcinogenic agent (6), which has already been demonstrated in hepatocellular carcinoma (18) and breast cancer (19). Divalent iron contributes to the formation of ROS, which may cause damage to the DNA resulting in mutations and the initiation of cancer growth. Iron is also required for cellular proliferation due to its role in the active sites of a wide range of proteins involved in energy metabolism, respiration and DNA synthesis.

The reduction in $\mathrm{T}_{2}$ (hypothetically caused by an increased iron concentration) in the BG and Th observed in the present study may be an indirect consequence of tumour development rather than direct damage. Direct damage to the $\mathrm{BG}$ tissue by the active tumour is improbable; reduced $\mathrm{T}_{2}$ values contralateral to the tumour were observed and no changes were detected in the WM in the case of untreated tumours, although the majority of the tumours had affected the WM. Lower $\mathrm{T}_{2}$ values in the WM of patients with recurrent tumours (not observed in the patients with newly diagnosed tumours) may be a consequence of the damage to the tissue due to the therapy undergone. The observed changes in the BG are possibly the result of a general systemic effect of the tumours. 
Although the concentration of the iron-storage protein ferritin in CSF in patients with glioblastoma has been found to be high, as compared with controls (11), the contribution of ferritin to $\mathrm{T}_{2}$ shortening may be disregarded. The reported mean concentration of ferritin in the CSF was $103 \mathrm{ng} / \mathrm{ml}(10.3 \mu \mathrm{g} / 100 \mathrm{~g})(11)$, which is negligible, as compared with the concentration of non-haem iron in the $\mathrm{BG}$, as reported in another study $(21.3 \mathrm{mg} / 100 \mathrm{~g}$ fresh weight in the GP), the $\mathrm{Th}(4.76 \mathrm{mg} / 100 \mathrm{~g}$ fresh weight) or even the frontal WM (4.24 mg/100 g) (1).

The key issue is the transport of the putative iron into the $\mathrm{BG}$ or Th. In the present study, no signal enhancement to the contrast-enhanced MR images was observed in the BG; therefore, the blood brain barrier was presumed to be intact. Thus, the direct transport of ferritin molecules between the plasma and the tissue is improbable. However, previously identified elevated ferritin concentrations in the CSF indicate that the CSF may be an alternative route for iron transportation to the brain tissue (11).

As the tumour may be responsible for increased iron concentrations in the plasma (17), the transport may also be corrupted at the transferrin/transferrin receptor level. An increase in the concentration of ferrous ions inside the cell indicates the threat of oxidation stress induced by $\mathrm{Fe}^{2+}$. To protect the tissue, the ferrous ions are trapped and oxidised to ferric ions within the ferritin molecules and stored. This accelerated protective process may result in a substantially higher deposition of iron compounds in the BG or Th. This hypothesis is in accordance with the known role of iron in carcinogenesis and the finding that the maintenance of iron homeostasis by chelation slows tumour growth (19).

Notably, in the present study, similarly reduced $\mathrm{T}_{2}$ values were detected in the two patients with Lym and the patients with gliomas (Fig. 2), although the origin of Lym tumours is in the lymphatic tissue, in contrast to gliomas, which are intrinsic to the brain tissue. This finding corresponds to the hypothesis that increased iron accumulation is a reaction to localised alterations in iron homeostasis associated with tumour growth and angiogenesis rather than a consequence of direct changes in the brain tissue.

The present study demonstrated that the reduction in the $\mathrm{T}_{2}$ relaxation time in patients with brain tumours was possibly caused by the deposition of paramagnetic ions in the BG and Th. Non-specific changes in the $\mathrm{T}_{2}$ relaxation times in the $\mathrm{BG}$ occurred in patients with untreated tumours and those with recurrent tumours, regardless of tumour localisation. We hypothesise that the changes in the BG and Th are caused by iron deposition in ferritin molecules to eliminate excessive ferrous ions from the tissue in order to provide protection from oxidative stress.

Although the present study has no direct impact on current diagnosis or tumour treatment practices, the study contributes to the understanding of how brain tumours influence, through homeostatic changes, even distant healthy tissues.

\section{Acknowledgements}

This study was supported by the Ministry of Health, Czech Republic-Conceptual Development of Research Organisation (Institute for Clinical and Experimental Medicine-IKEM; IN 00023001).

\section{References}

1. Hallgren B and Sourander P: The effect of age on the non-haemin iron in the human brain. J Neurochem 3: 41-51, 1958.

2. Vymazal J, Babis M, Brooks RA, Filip K, Dezortova M, Hrncarkova H and Hajek M: T1 and T2 alterations in the brains of patients with hepatic cirrhosis. AJNR Am J Neuroradiol 17: 333-336, 1996.

3. Wolozin B and Golts N: Iron and Parkinson's disease. Neuroscientist 8: 22-32, 2002.

4. Correia S, Hubbard E, Hassenstab J, et al: Basal ganglia MR relaxometry in obsessive-compulsive disorder: T2 depends upon age of symptom onset. Brain Imaging Behav 4: 35-45, 2010.

5. Hájek M, Adamovičová M, Herynek V, Škoch A, Jírů F, Krepelová A and Dezortová M: MR relaxometry and $1 \mathrm{H}$ MR spectroscopy for the determination of iron and metabolite concentrations in PKAN patients. Eur Radiol 15: 1060-1068, 2005.

6. Toyokuni S: Iron-induced carcinogenesis: The role of redox regulation. Free Radic Biol Med 20: 553-566, 1996.

7. Evans AE, D'Angio GJ, Propert K, Anderson J and Hann HW: Prognostic factor in neuroblastoma. Cancer 59: 1853-1859, 1987.

8. Potaznik D, Groshen S, Miller D, Bagin R, Bhalla R, Schwartz M and de Sousa M: Association of serum iron, serum transferrin saturation and serum ferritin with survival in acute lymphocytic-leukemia. Am J Pediat Hematol Oncol 9: 350-355, 1987.

9. Richmond HG: Induction of sarcoma in the rat by iron-dextran complex. Br Med J 1: 947-949, 1959.

10. Huang X: Iron overload and its association with cancer risk in humans: evidence for iron as a carcinogenic metal. Mutat Res 533: 153-171, 2003.

11. Sato Y, Honda Y, Asoh T, Oizumi K, Ohshima Y and Honda E: Cerebrospinal fluid ferritin in glioblastoma: evidence for tumor synthesis. J Neurooncol 40: 47-50, 1998.

12. Richardson DR, Kalinowski DS, Lau S, Jansson PJ and Lovejoy DB: Cancer cell iron metabolism and the development of potent iron chelators as anti-tumour agents. Biochim Biophys Acta 1790: 702-717, 2009.

13. Vymazal J, Urgosík D and Bulte JW: Differentiation between hemosiderin- and ferritin-bound brain iron using nuclear magnetic resonance and magnetic resonance imaging. Cell Mol Biol (Noisy-le-grand) 46: 835-842, 2000.

14. Schenker C, Meier D, Wichmann W, Boesiger P and Valavanis A: Age distribution and iron dependency of the T2 relaxation time in the globus pallidus and putamen. Neuroradiology 35: 119-124, 1993.

15. Herynek V, Wagnerová D, Hejlová I, Dezortová M and Hájek M: Changes in the brain during long-term follow-up after liver transplantation. J Magn Reson Imaging 35: 1332-1337, 2012.

16. Dusek P, Jankovic J and Le W: Iron dysregulation in movement disorders. Neurobiol Dis 46: 1-18, 2012.

17. Steegmann-Olmedillas JL: The role of iron in tumour cell proliferation. Clin Transl Oncol 13: 71-76, 2011.

18. Deugnier Y: Iron and liver cancer. Alcohol 30: 145-150, 2003.

19. Jian J, Yang Q, Dai J, Eckard J, Axelrod D, Smith J and Huang X: Effects of iron deficiency and iron overload on angiogenesis and oxidative stress - a potential dual role for iron in breast cancer. Free Radic Biol Med 50: 841-847, 2011.

20. Richardson DR: Iron chelators as therapeutic agents for the treatment of cancer. Crit Rev Oncol Hematol 42: 267-281, 2002. 